\title{
The grounding argument against non-reductive moral realism
}

\author{
Ralf M. Bader \\ Merton College, University of Oxford
}

ABSTRACT: The supervenience argument against non-reductive moral realism threatens to rule out the existence of irreducibly normative properties by establishing that for every normative property there is a corresponding non-normative property that is necessarily co-extensive with it. This paper first considers but rejects the suggestion that one can address the supervenience argument by insisting that normative properties only supervene with normative but not metaphysical necessity. It then establishes that the standard response to the supervenience argument, which consists in rejecting necessary co-extension as the criterion for property identity, does not suffice for defending non-reductionist views of the normative. In particular, it identifies a hyperintensional analogue of the supervenience argument that threatens non-reductionism even within a hyperintensional setting. This grounding argument against non-reductive moral realism threatens to rule out the existence of irreducibly normative properties by establishing that for every normative property there is a corresponding non-normative property that has the very same grounds and is, accordingly, hyperintensionally equivalent. It is then argued that non-reductionism can nevertheless be salvaged by distinguishing the different grounding relations that are involved in grounding the normative property and the corresponding non-normative property. Nonreductionist versions of moral realism thus turn out to be committed to there being irreducibly different grounding relations. 


\section{The supervenience argument}

Strong supervenience of normative properties on non-normative properties implies that for every normative property there is a necessarily co-extensive nonnormative property, given that the set of non-normative properties is closed under infinitary disjunction and conjunction (cf. Kim: 1993, chapter 3; Jackson: I998; Streumer: 2008). ${ }^{\mathrm{I}, 2}$ This puts pressure on non-reductionist versions of moral realism, insofar as normative properties would seem to end up being identical to non-normative properties, thereby ruling out the existence of irreducibly normative properties. ${ }^{3,4}$

I. normative properties strongly supervene on non-normative properties

2. the set of non-normative properties is closed under infinitary disjunction and conjunction

$\therefore$ for every normative property there is an intensionally equivalent nonnormative property

3. intensionally equivalent properties are identical

$\therefore$ every normative property is identical to a non-normative property

\section{Normative supervenience}

Premise I can be construed in two ways, namely as positing either a normative or a metaphysical supervenience relation.

I-NORM: 'normative properties strongly supervene with normative necessity on non-normative properties'.

I-META: 'normative properties strongly supervene with metaphysical necessity on non-normative properties'.

${ }^{1}$ Kim's proof appealed to B-maximal properties that presuppose infinitary Boolean closure, which is problematic since closure under complementation is implausible for the set of nonnormative properties and cannot be assumed without begging the question against the proponent of irreducibly normative properties. Yet, van Cleve showed that Kim's result can be established by means of fewer resources. Since we only need to appeal to B-natures and not to B-maximal properties, closure under infinitary disjunction and conjunction is sufficient (cf. van Cleve: 1990).

${ }^{2}$ When concerned with the supervenience of the normative on the non-normative, one is primarily concerned with the instantiation of normative properties (e.g. a particular value bearer, say action $a$, instantiates the normative property of being good), i.e. with particular facts rather than with general facts such as $\phi$-ing is good.

${ }^{3}$ As Streumer has pointed out, this terminology may be somewhat confusing, given that a reductionist view will be categorised as one identifying normative properties with non-normative properties (cf. Streumer: 20I3, p. 3 I2). Non-normative properties are thus to be understood not negatively as not being normative properties, but positively as being descriptive properties.

${ }^{4}$ Analogous arguments can be run against irreducibly normative facts or truths. 
Defenders of irreducibly normative properties can challenge the supervenience argument on the basis that I-META is required for the argument to succeed but that only the weaker I-NORM is warranted.

If normative properties only normatively supervene on non-normative properties, such that the supervenience claim only holds with normative but not metaphysical necessity, then normative properties will only be necessarily co-extensive with their non-normative counterparts when it comes to normative modality. For each normative property there will be a non-normative property that has the same extension in all normatively possible worlds. This, however, does not suffice to establish the conclusion that normative properties are identical to non-normative properties. Metaphysical necessity is required if necessarily co-extensive properties are to be identical, given that the identity and distinctness of properties is a metaphysical matter.

When only accepting I-NORM, the relation between normative and nonnormative properties is analogous to that between dispositional and categorical properties (for those rejecting dispositional essentialism). Dispositional properties strongly supervene on categorical properties. Strong supervenience implies the possibility of constructing for every dispositional property a necessarily coextensive disjunctive property formed out of categorical properties by suitably conjoining and disjoining the categorical properties that form the supervenience bases of dispositional properties. Since categorical properties satisfy the relevant closure conditions, insofar as a disjunction of conjunctions of categorical properties is itself a categorical property, it would seem that one ends up being committed to dispositional properties being identical to categorical properties.

This conclusion, however, is misguided since identity requires (at a minimum) necessary co-extensiveness across all metaphysically possible worlds. The supervenience of dispositional on categorical properties, however, only holds with nomological necessity. The same categorical properties can give rise to different dispositions if the causal laws are different, such that a duplicate of a fragile object in a world with different laws need not also be fragile. The laws make a difference and thereby ensure that the supervenience claim involves the wrong modality for establishing reductionist conclusions.

By insisting on a normative rather than metaphysical supervenience claim, one would thus seem to be able to defend the irreducibility of normative properties in a manner that is analogous to how dispositional properties turn out to be distinct from and not reducible to categorical properties, given that they only supervene nomologically but not metaphysically.

Streumer has claimed that defending irreducibly normative properties by appealing to normative supervenience implies the objectionable commitment that "it is possible for an object to gain an additional normative property without this object or any other object gaining any additional descriptive property" (Streumer: 2008, p. 559). Merely having normative but not metaphysical supervenience 
would, in this way, seem to allow for objectionably brute and unexplained variation in normative properties.

Whilst a brute difference in normative properties would indeed be problematic, normative supervenience allows us to invoke differences in normative laws in order to explain variation in normative properties. If $\mathrm{x}$ in $\mathrm{w}_{\mathrm{I}}$ has normative property $\mathrm{N}_{1}$ whilst $\mathrm{y}$ in $\mathrm{w}_{2}$ has some other normative property $\mathrm{N}_{2}$, even though these worlds are completely indiscernible in terms of descriptive properties, then this difference is not brute but unproblematic as long as this variation can be explained in terms of normative law $\mathrm{L}_{\mathrm{I}}$ holding in $\mathrm{w}_{\mathrm{I}}$, whilst a different law $\mathrm{L}_{2}$ holds in $\mathrm{w}_{2}$. A failure of metaphysical supervenience, whereby one ends up countenancing a difference in properties that is unexplained and brute, differs importantly from a case in which one can appeal to the normative laws to explain the differences in normative properties.

Although the differences in laws themselves will be brute, this is unproblematic since the non-reductive realist can consider these laws to be fundamental and irreducible. ${ }^{5}$ When construed as robust principles that govern grounding relations, they are to be understood as basic and not in need of explanation. ${ }^{6}$ This means that, whereas normative properties are derivative properties and hence are such that something is required to account for differences in these properties, no underlying difference is required to account for differences in normative laws. ${ }^{7}$

A related objection to the idea that normative properties might only supervene with normative necessity is that "it seems impossible that another world might be identical to this one except that in that other world, a genocide otherwise identical to the actual Rwandan genocide differed solely in being ethically wonderful, rather than being an atrocity" (McPherson: 20I2, p. 212). The thought is that a supervenience claim with merely normative modal force only makes such objectionable scenarios normatively impossible, since they are incompatible with the actual normative laws, but allows for these scenarios to be metaphysically possible.

The suggestion that metaphysical supervenience is required to preclude the possibility of such objectionable scenarios is, however, problematic. This is because the rejection of metaphysical necessity does not imply that anything goes. Accepting that normative principles can be metaphysically contingent is not to say that anything could have been good or right, in the same way that saying that

\footnotetext{
${ }^{5}$ Normative laws are not fundamental in the sense of occupying the basic level of a grounding hierarchy, but rather in the sense of standing outside this hierarchy and inducing its structure.

${ }^{6}$ If the laws themselves were to supervene on the descriptive facts, then it would not be possible for worlds that are indiscernible in terms of non-normative properties to differ in terms of laws. Likewise if the laws were merely to describe and summarise grounding relations, rather than governing them, then a difference in laws would presuppose rather than explain a difference in normative properties, thereby rendering normative variation across descriptively indiscernible worlds a brute fact.

${ }^{7}$ Analogously, differences in dispositional properties amongst objects that have the same categorical properties can be explained in terms of differences in causal laws, whereas differences in causal laws will be basic (for those who consider them to be robust governing laws).
} 
the causal laws could have been different does not imply that anything could have caused anything (or for that matter that there could be uncaused events).

In fact, there can be robust constraints (such as consistency and universalisability requirements) on what the normative laws can be like, in the same way as there are substantive constraints on what systems of causal laws can be like. For instance, whilst it may be contingent what normative laws obtain, it may be necessary that some normative laws or other do obtain. That is, it might be necessary that there are laws without there being any necessary laws, thereby making it metaphysically impossible for there to be amoral worlds (in the same way as it may be metaphysically impossible for there to be acausal worlds). These constraints will hold with metaphysical necessity and will delimit the range of possible normative laws, potentially allowing one to rule out the objectionable scenario envisaged by McPherson. ${ }^{8}$

The real problem with addressing the supervenience argument by invoking normative supervenience is rather that normative modality might, for all we know, coincide with metaphysical modality. ${ }^{9}$ The set of normatively possible worlds might turn out to be the same as the set of metaphysically possible worlds, in which case even normative supervenience would imply the existence of nonnormative properties that are metaphysically necessarily co-extensive with normative properties. This happens on views that countenance a unique set of normative principles that hold in all metaphysically possible worlds. ${ }^{\text {Io }}$ On such views, normative supervenience will suffice for necessary co-extensiveness with respect to metaphysical modality and hence would seem to preclude the existence of irreducibly normative properties.

In fact, the most plausible forms of moral realism do not allow for variation in normative laws. If normative laws were to be metaphysically contingent, then very serious epistemological concerns would arise. Contingency amounts to there being a range of systems of laws that hold in different regions of modal space. The problem then is to identify the laws that hold in the actual world. Unlike in the case of causation, where we experience the things standing in causal relations and thereby can identify patterns of interaction, we cannot identify normative laws a posteriori. Since normative facts are metaphysically independent of non-normative facts according to the contingency proposal, which means that

\footnotetext{
${ }^{8}$ So far, all that has been shown is that the possibility of these scenarios might be ruled out, and that a denial of metaphysical supervenience, accordingly, does not imply a commitment to their possibility. A fully satisfactory reply would require one to specify the precise nature of these constraints, showing how exactly they manage to preclude objectionable scenarios.

${ }^{9}$ This (epistemic) possibility cannot be ruled out even by those who consider normative necessity to be an irreducible necessity (pace Fine: 2005, p. 252).

Io These principles have, what Enoch calls, modally maximal jurisdiction (cf. Enoch: 20I I, p. I45). Whether normative principles do vary across modal space is not a normative but a metaphysical matter (contra Scanlon: 20I4, p. 4I), i.e. the scope of these principles is a function of the metaphysical status of their source.
} 
descriptively indistinguishable worlds can differ with respect to normative laws and hence differ in terms of normative facts, there is no way to identify them on the basis of non-normative facts. One would thus need some form of direct access to the normative facts, for instance via a quasi-perceptual faculty. Not only do we lack such a faculty, this would imply an implausible methodology for ethics, namely a scientific methodology based on gathering data and identifying patterns. Nor can the normative laws be identified a priori since the contingency of the laws ensures that it is a brute fact as to which laws happen to obtain in the actual world that cannot be discerned by reasoning. Since the laws are brute and the evaluative properties the grounding of which they govern are unobservable, there does not seem to be any way for us to identify them. This means that, even if one can identify a priori the range of possible systems of normative laws, one cannot identify which ones obtain in the actual world. All that can be identified is the invariant core of these possible systems. Yet, this core may very well be empty and any differences beyond the core are brute differences that are not epistemically accessible to us. ${ }^{\text {II }}$

Moreover, invoking normative supervenience to defend irreducibility does not adequately capture the difference and heterogeneity between normative and non-normative properties. The distinctness of these properties should not be contingent on how normative modality is related to metaphysical modality (i.e. it should not be dependent on the modal status of the normative laws). A theory that has to rely on the normatively possible worlds forming a proper subset of those that are metaphysically possible does not adequately respect and capture the fact that these properties are different in kind and do not merely differ in extension across modal space. In short, it is the nature of these properties that should account for their distinctness (and that should also explain any differences in extensions across possible worlds), not the relation between normatively and metaphysically possible worlds.

Accordingly, one needs an alternative theory that is hyperintensional in character, that generates a robust difference in kind between normative and nonnormative properties, and that does not require normative modality to be restricted, in the sense of the normatively possible worlds forming a proper subset of the metaphysically possible worlds. ${ }^{\text {I2 }}$

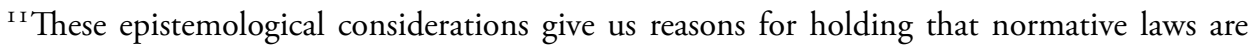
invariant across modal space, that morality is not a brute phenomenon that just happens to be a certain way in the actual world, but that there are reasons for it being the way it actually is and that its laws are intelligible and can be identified by reasoning. Unfortunately, they do not constitute reasons as to why this is the case and realists will need to provide an explanation for there being a unique set of principles, ideally by identifying a modally invariant source. This explanation will have to account for the disanalogy between causation and morality, explaining why there is co-extensiveness of metaphysical and normative possibility in the moral case, even though there is no analogous co-extensiveness in the causal case.

${ }^{12}$ As we will see later on, it turns out that the very same commitments that a realist requires in
} 
An analogous problem arises in the case of dispositions when nomological possibility is taken to coincide with metaphysical possibility. Views that consider nomological possibility to be co-extensive with metaphysical possibility (such as certain versions of dispositional essentialism) will not be able to distinguish dispositional properties from their corresponding disjunctive categorical counterparts in terms of their extensions across modal space. The nomological principles connecting dispositional properties to their categorical bases will then hold in the same set of circumstances as the metaphysical principles connecting disjunctive properties to their disjuncts.

This is problematic since the difference between dispositional and categorical properties is meant to be a difference in kind that should not depend on the relation between these two types of modality (nor on the status of the causal laws). In order to avoid reducibility and preserve the idea that categorical and dispositional properties are different in kind, one should not rely on the idea that the different modal strengths that are involved in nomological and metaphysical supervenience lead to different extensions across modal space. Instead, one has to bring in hyperintensional resources.

\section{Hyperintensional differences}

Although it is possible to deny necessary co-extensiveness with respect to metaphysical modality by arguing for a restricted normative supervenience claim, this response does not really get to the heart of the matter. Accordingly, it is preferable to find a response that succeeds even if metaphysically necessary co-extensiveness is granted.

A plausible alternative consists in rejecting premise 3 by denying that necessarily co-extensive properties have to be identical. The suggestion is that nonreductionism can be salvaged by rejecting the idea that intensional equivalence, i.e. having the same extension across modal space, suffices for identity (cf. ShaferLandau: 2003; Majors: 2005; Suikkanen: 2010). Instead of individuating properties intensionally, one adopts a hyperintensional account that allows necessarily co-extensive properties to be distinct. This would seem to avoid the commitment to reducibility by opening up room for the possibility that normative properties are distinct from non-normative properties, despite being necessarily co-extensive. In the same way that necessarily co-extensive properties, such as triangularity and trilaterality, can be distinct, so can normative properties be distinct from their non-normative disjunctive counterparts. This is the standard reply to

order to justify scope restrictions (i.e. consider laws as holding in a restricted set of metaphysically possible worlds) also allow us to address the case where normative supervenience goes together with metaphysically necessary co-extensiveness. The restriction strategy thus presupposes the very same hyperintensional resources that are employed in the defence of irreducibly normative properties that is provided later on and that does not require or involve any restrictions. 
the supervenience argument.

Simply denying that all necessarily co-extensive properties are identical and saying that property identity is a hyperintensional matter, however, leaves much open. Though opening up room for the distinctness of normative properties from descriptive properties, it is far from being sufficient for a full-fledged defence of irreducibly normative properties. In order for this line of response to be substantiated, one needs to provide a fine-grained theory of properties that specifies the identity conditions of properties and that explains how hyperintensional differences can arise. What is required is a satisfactory account of the identity conditions of properties that explains which necessarily co-extensive properties are identical and which ones distinct.

The proposed theory has to do two things if it is to salvage the idea of irreducibly normative properties. First, it needs to establish distinctness. One has to show that normative properties and their descriptive disjunctive counterparts in fact turn out to be distinct on the proposed theory. In this way, one can explain what accounts for their distinctness and what thereby distinguishes these properties from other 'pairs' of necessarily co-extensive properties that are identical. Second, it needs to establish a difference in kind. Not only do normative and non-normative properties have to be distinct, they also have to differ in kind. This means that the proposed theory has to account for the differences between these properties and explain how it is that they differ in kind, i.e. why one is normative whereas the other is non-normative despite being so intimately connected (in particular, despite being necessarily co-extensive and, as we will see later, despite having the very same grounds).

Moreover, in order for such a theory to be satisfactory, it has to satisfy the requirement (which we can call the 'worldliness constraint') that it tracks worldly differences, rather than simply identifying differences in how we pick out properties and represent the world. The differences that it posits amongst properties must be ontologically robust. As Jackson notes, the account has to ensure that a property is "an aspect of the world, not an aspect of our discourse or thought about it" (Jackson: I998, p. I26). Otherwise, we will not end up with a real distinction but with a merely nominal distinction. For instance, a satisfactory theory needs to yield the verdicts that $(F \wedge G)$ is identical to $(G \wedge F)$, that $\neg \neg \neg F$ is identical to $\neg \mathrm{F}$, and that $((\mathrm{F} \wedge \mathrm{G}) \wedge \mathrm{H})$ is identical to $(\mathrm{F} \wedge(\mathrm{G} \wedge \mathrm{H}))$ as well as to the ternary conjunction $\bigwedge\{\mathrm{F}, \mathrm{G}, \mathrm{H}\}$. Any theory that treats such properties as distinct draws invidious distinctions and cannot claim to be tracking worldly differences.

This means that the theory needs to be fine-grained without being too finegrained. Theories of property individuation form a spectrum. At one end of the spectrum are ultra coarse-grained theories that collapse all distinctions amongst properties, whereas at the other end of the spectrum are ultra fine-grained theories, according to which every way of picking out a property corresponds to a 
distinct property. What is required is a systematic and principled account that is more fine-grained than intensional equivalence without being too fine-grained. Otherwise one will no longer be in the realm of the worldly but in the realm of the conceptual, identifying merely nominal rather than real differences. In short, one needs a worldly notion of hyperintensional equivalence.

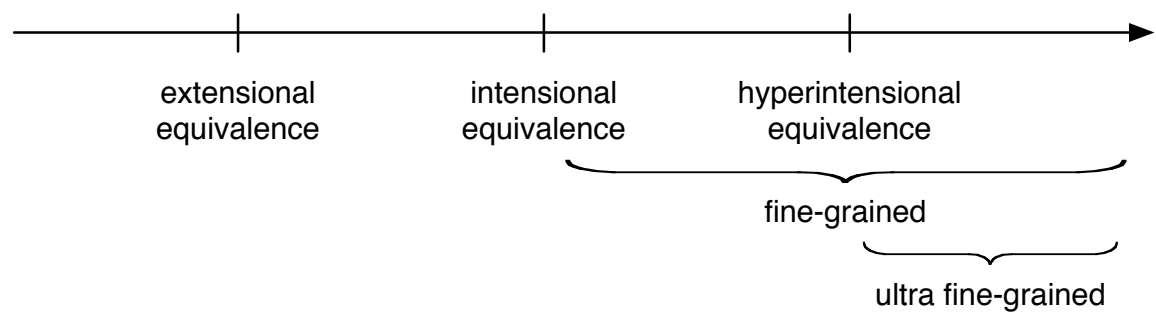

The worldliness constraint can be satisfied by individuating properties in terms of their basic grounds. Given the set of fundamental properties $\mathcal{F}$ that contains all those properties that are ungrounded, we can construct the set of basic grounders $\mathcal{B}$, which consists of all compatible pluralities (including degenerate pluralities) of fundamental properties. The grounding set $\mathrm{g}(\mathrm{F})$ of a property $\mathrm{F}$ is that set that contains all basic proper and improper grounders of $\mathrm{F}$, i.e. $\mathrm{g}(\mathrm{F})=\{\Gamma \in \mathcal{B}: \Gamma$ grounds $\mathrm{F}\}$. Two (non-fundamental) properties $\mathrm{F}$ and $\mathrm{G}$ are then identical iff they are hyperintensionally equivalent, i.e. $g(F)=g(G) .{ }^{13}$

One thereby recognises that properties can be had in different ways, assessing not only whether a property is had but also how it is had. One moves away from an extensional understanding that only considers whether a property is had to a hyperintensional account that is also concerned with the ways in which a property is had. Property identity then requires properties to be had in the very same ways, which allows there to be properties that are instantiated by the same possibilia but that can be distinct, given that they are had in different ways due to being grounded differently. Such a theory provides a more fine-grained individuation than necessary co-extension, whilst identifying worldly differences insofar as the distinctness of properties is explained in terms of there being different features of the world that account for their instantiations. As a result, we are not merely dealing with "a separation in modes of representation in thought" (Jackson: I998, p. 26) but with a genuine separation in reality.

It might then be thought that the crucial step of the supervenience argument, that moves from necessary co-extension to identity, can be rejected by requiring properties to be not only intensionally but also hyperintensionally equivalent and that normative properties can, accordingly, be distinguished from non-normative properties, thereby avoiding a commitment to reductionism.

\footnotetext{
${ }^{13}$ The resulting hyperintensional logic is developed in "Hyperintensional equivalence" (Bader: manuscript).
} 


\section{The grounding argument}

Rejecting necessary co-extension as the criterion for property identity, however, does not suffice for defending the irreducibility of the normative. This is because there is an analogous grounding argument that functions as the hyperintensional analogue of the supervenience argument.

$I^{\prime}$. normative properties are grounded in non-normative properties

$2^{\prime}$. the set of non-normative properties is closed under infinitary disjunction and conjunction

$3^{\prime}$. disjunctive properties are (individually) grounded in their disjuncts ${ }^{14}$

$4^{\prime}$. conjunctive properties are (collectively) grounded in their conjuncts

$\therefore$ for every normative property there is a hyperintensionally equivalent non-normative property

$5^{\prime}$. hyperintensionally equivalent properties are identical

$\therefore \quad$ every normative property is identical to a non-normative property

If normative properties do not only supervene on non-normative properties but are grounded therein, then for every normative property $\mathrm{N}$ there is a disjunctive descriptive property $\mathrm{D}$ that is grounded in precisely the same non-normative properties as $\mathrm{N}$, thereby threatening non-reductionism even within a hyperintensional setting. Merely claiming that properties can be distinct despite being necessarily co-extensive thus does not suffice for defending irreducibility.

Premise $\mathrm{I}^{\prime}$ holds that normative properties are grounded in descriptive properties in the sense that whenever a normative property $\mathrm{N}$ is instantiated there are certain descriptive properties in virtue of which $\mathrm{N}$ is instantiated. This strengthening of the claim that normative properties strongly supervene should be unproblematic, given that grounding relations are precisely the kinds of explanatory relations that supervenience relations are meant to model and in terms of which supervenience claims can ultimately be explained and justified.

On the one hand, the motivations and intuitions cited in favour of supervenience usually take the form of resultance intuitions, whereby instantiations of normative properties are taken to be explained by instantiations of non-normative properties. Properly capturing these intuitions requires not only accepting the dependent-variation of normative on non-normative properties that is encapsulated by a supervenience claim, but a commitment to a grounding relation connecting these properties.

On the other hand, even those motivated by the thought that certain facts simply could not have failed to have the normative status that they do have (e.g. that genocide is wrong, cf. McPherson: 2012, p. 212) naturally end up endorsing a grounding claim. This is because accepting a supervenience claim engen-

\footnotetext{
${ }^{14}$ If the disjuncts are compatible, then the disjunction can also be collectively grounded in them taken together.
} 
ders an explanatory burden that needs to be discharged. This burden is particularly pressing, given the heterogeneity of normative and non-normative properties endorsed by the non-reductive realist. Unless there is some dependence relation between these properties, it would seem that they can be independently recombined, which would undermine the supervenience claim. The necessary dependent-variation should, accordingly, not be brute but explicable.

This can be achieved by positing a grounding relation. This ensures that the properties cannot be independently recombined but that there is dependentvariation of the grounded properties on their grounds. A grounding relation explains why that which is dependent, namely the normative, varies with that on which it depends, namely the non-normative. The grounding of normative in non-normative properties implies the supervenience of the former on the latter, thereby allowing us to discharge the explanatory burden that is incurred when positing the supervenience of the normative. The supervening set consists of normative properties $N_{I} \ldots N_{n}$. Each such property $N_{i}$ has instantiations $n_{i}^{I} \ldots n_{i}^{n}$, whereby every instance $n_{i}^{j}$ will be grounded in a collection of instantiations of descriptive properties $D_{I} \ldots D_{n}$, such that the subvening set of base properties will consist of the union of these collections of descriptive properties for all instances of all normative properties. ${ }^{\text {IS }}$ The fact that objects that do not differ non-normatively cannot differ normatively is then explained on the basis that normative properties are resultant properties that are grounded in non-normative properties. This means that denying premise $I^{\prime}$ either leads to an objectionable commitment to brute necessary connections or alternatively to an implausible rejection of strong supervenience.

It might be suggested that premise $\mathrm{I}^{\prime}$ only appears innocuous because of an equivocation. Given that grounding can be either full or partial, there are two construals of this premise.

I'-FULL: 'normative properties are fully grounded in non-normative properties'.

I'-PARTIAL: 'normative properties are partially grounded in non-normative properties'.

The grounding argument presupposes the former reading. In order for $\mathrm{N}$ to have the very same grounds as $\mathrm{D}, \mathrm{N}$ has to be fully grounded in the relevant nonnormative properties out of which $\mathrm{D}$ is constructed. Otherwise, the grounding set of $\mathrm{D}$ would only be a proper subset of that of $\mathrm{N}$, in which case $\mathrm{D}$ would not be identical to $\mathrm{N}$ but would rather be a (non-basic) ground of $\mathrm{N}$. It might now be objected that normative properties are only partially grounded in nonnormative properties since they are also partially grounded in normative laws, i.e. that non-normative properties only ground normative properties together with

\footnotetext{
${ }^{15}$ For some types of supervenience claims, the base will consist of the Boolean closure of this set rather than of the set itself.
} 
the normative laws. On such a view, normative properties have different grounds than their descriptive disjunctive counterparts because the former are not only grounded in non-normative properties but also in laws.

However, to think of the laws as being grounds involves a confusion of levels. ${ }^{16}$ One has to distinguish good-makers (i.e. grounds) from makers of goodmakers (i.e. grounding principles). Including the laws amongst the grounds is analogous to including inference rules amongst the premises, yet we do not infer q from: 1 . p, 2. if $\mathrm{p}$ then $\mathrm{q}$, and 3. modus ponens, but rather infer $\mathrm{q}$ via modus ponens from the two premises: $\mathrm{I}$. p, and 2. if $\mathrm{p}$ then $\mathrm{q}$. Rather than themselves being premises, the inference rules connect the premises to the conclusion. Likewise, it is analogous to considering laws of nature to be partial causes, which is confused since the breaking of the window is not caused by the causal laws together with the throwing of the stone. Instead, the laws govern the causal relations between causes and effects. Normative laws, inference rules, and causal laws all play an important role, yet one that is distinct from that played by grounds, premises, and causes.

Since normative laws do not ground normative properties but govern the grounding relations connecting non-normative grounds to normative properties, they are not to be included amongst the grounds but amongst the grounding principles. Rather than normative properties being metaphysically grounded in non-normative properties together with the normative laws, they are normatively grounded in the non-normative properties via these laws. Put differently, they modify the grounding relation rather than featuring as relata. There is hence no difference in terms of what these normative and descriptive properties are grounded in. Instead, as we will see in section 6, there is only a difference in terms of how they are grounded in the base that they share.

Premise $2^{\prime}$ is unchanged. This premise is very plausible, since it is difficult to see how conjoining and disjoining non-normative properties could ensure that the resulting property would fail to be non-normative. At any rate, the proponent of irreducibly normative properties (to whom this argument is addressed) should have no qualms with this premise, given that he considers normative and non-normative properties to differ in kind. Such a difference in kind cannot be straddled by simply conjoining or disjoining non-normative properties. ${ }^{17}$

Premises $3^{\prime}$ and $4^{\prime}$ are likewise uncontroversial. The grounding of disjunctive and conjunctive properties in their constituents is usually considered to be a paradigm case of grounding. (Whilst these premises themselves are uncontroversial, certain difficulties arise when employing them in constructing hyperintensionally equivalent properties, as we will see in sections 7 and 8.)

These premises imply that for every normative property, there is a descriptive

\footnotetext{
${ }^{16}$ Cf. "Two levels of good-making” (Bader: manuscript).

${ }^{17}$ The fact that the relevant closure conditions require infinitary property-forming operations is unproblematic, cf. Bader: $20 \mathrm{I} 2$ contra Glanzberg: $200 \mathrm{I}$.
} 
property that has the very same grounds. This is because every (actual as well as possible) instantiation $n_{i}$ of a normative property $\mathrm{N}$ is grounded in a collection of instantiations of non-normative properties $\Gamma_{i}$. All the different pluralities $\Gamma_{\mathrm{I}} \ldots \Gamma_{\mathrm{n}}$ that ground instantiations $\mathrm{n}_{\mathrm{I}} \ldots \mathrm{n}_{\mathrm{n}}$ together constitute the basic grounders of $\mathrm{N}$, i.e. the members of N's grounding set $\mathrm{g}(\mathrm{N})$. Out of these pluralities we can construct a disjunctive descriptive property $\mathrm{D}$ that has the very same grounds as $\mathrm{N}$ by disjoining the conjunctive properties that are formed by conjoining the members of $\Gamma_{i}$ for every possible instantiation $n_{i}$ of $N$. More precisely, for any $\Gamma_{\mathrm{i}}$ we form a conjunctive property $\wedge \Gamma_{\mathrm{i}}$ (which equals $F_{\mathrm{I}} \wedge \mathrm{F}_{2} \ldots \wedge \mathrm{F}_{n}$ for all $F_{\mathrm{i}}$ that are amongst $\Gamma_{\mathrm{i}}$ ). By disjoining all such conjunctive properties one ends up with a property $\mathrm{D}=\bigvee\left\{\wedge \Gamma_{\mathrm{i}}\right.$ for all $\left.\Gamma_{\mathrm{i}} \in \mathrm{g}(\mathrm{N})\right\}$ that is not only necessarily co-extensive with $\mathrm{N}$ but that also has the very same grounds, since both have $\Gamma_{\mathrm{I}} \ldots \Gamma_{\mathrm{n}}$ as their basic grounders, i.e. $\mathrm{g}(\mathrm{D})=\mathrm{g}(\mathrm{N}) .^{\mathrm{I}}$

Given the closure of non-normative properties under infinitary conjunction and disjunction, this implies that the disjunctive property $\mathrm{D}$ that is hyperintensionally equivalent to a particular normative property $\mathrm{N}$ will be a non-normative property. And given that hyperintensionally equivalent properties are identical, normative properties then turn out to be identical to non-normative properties.

Claiming that properties are distinct becomes problematic when they are not only had by the same possibilia but are also such that the very same facts explain why these properties are had. Since there does not seem to be anything in the world that can render them distinct, it is rather difficult to account for their distinctness in a way that satisfies the worldliness constraint, such that we do not merely end up with a distinction at the level of description or representation but with a genuine difference in the world.

Critics of the supervenience argument frequently appeal to the (supposed) distinctness of triangularity and trilaterality and suggest that the distinctness of normative properties and their disjunctive counterparts can be understood along the same lines. The fact that triangularity and trilaterality are distinct properties can, however, be explained in terms of these properties having different grounds. The former property is had in virtue of having three angles, whereas the latter is had in virtue of having three sides. Different features of the world account for and give rise to these properties, thereby rendering them distinct.

\footnotetext{
${ }^{18}$ The disjunctive property formed in this way need not be identical to the disjunctive property formed by disjoining the conjunctions of B-properties on which the normative property supervenes, even when making use of a minimal supervenience base that does not include B-maximal properties that correspond to B-descriptions of the whole world but that only characterise the object's B-nature whilst leaving out irrelevant information. These properties can diverge where a property is overgrounded and where one basic ground is part of another, as happens when absorption principles fail. This divergence arises because grounding is characterised by a relevance constraint, whereas supervenience is subject to a more restrictive minimality constraint. For instance, $F$ is distinct from $H={ }_{d f}(F \vee(F \wedge G))$, allowing for $F$ as well as the plurality $F \circ G$ to be in the grounding set of $\mathrm{H}$, yet only $\mathrm{F}$ is in the supervenience base, since $\mathrm{F} \circ \mathrm{G}$ satisfies the relevance condition but not the minimality constraint.
} 
Yet, no such explanation is forthcoming in the case of normative properties and their disjunctive descriptive counterparts. These properties have the same grounding sets. Whatever grounds the former also grounds the latter, and vice versa. There is no difference in terms of what grounds these properties, no difference in terms of the features of the world that explain and give rise to them. There would, accordingly, not seem to be any genuine worldly difference between them, but merely a difference in terms of how we conceptualise and represent them. These properties are not only intensionally but also hyperintensionally equivalent and thus seem to be identical.

Accordingly, it turns out that the problem runs much deeper and that simply adopting a fine-grained criterion for individuating properties does not suffice for addressing this challenge to non-reductionist forms of moral realism. To circumvent the grounding argument one not only has to deny that necessary co-extensiveness suffices for identity, but also deny premise $4^{\prime}$, namely that hyperintensional equivalence suffices for identity. Doing so, however, would saddle one with an ultra fine-grained theory of properties that has no longer any claim to identifying genuine differences in the world. This is because any difference more fine-grained than hyperintensional equivalence understood in terms of sameness of basic grounders would not seem to satisfy the worldliness constraint.

Moreover, any theory that distinguishes (non-fundamental) properties that do not differ in terms of how they are grounded conflicts with the idea that the identity conditions of non-basic entities are provided by and specified in terms of the basic entities on which they depend, in this case that non-fundamental properties are individuated in terms of the fundamental properties in which they are grounded. Derivative entities are to be understood in terms of the things from which they derive, i.e. to be a particular derivative entity just is to be that entity which derives in certain ways from certain non-derivative entities. Since the identity of derivative properties is fixed by the fundamental properties from which they derive, it follows that sameness of grounds implies sameness of properties.

Relatedly, what higher-order properties derivate entities have, as well as what grounding work they can perform, is fixed by the non-derivative entities from which they derive. This means that one cannot explain the distinctness of $\mathrm{N}$ and $\mathrm{D}$ by appealing to differences in terms of what higher-order properties they have, nor to differences in terms of what $\mathrm{N}$ and $\mathrm{D}$ can ground. Attempts to use Leibniz's Law applied to properties in order to establish their distinctness accordingly fail. ${ }^{19}$ Arguments to the effect that normative and non-normative properties differ in terms of their higher-order properties and are hence distinct face the problem that higher-order properties are determined either by the lower-order properties or by the grounds of those properties. This, in turn, implies that any normative

${ }^{19}$ This kind of Leibniz's Law argument has been developed by Suikkanen: 2010, section 6 . Similarly, Enoch appeals to the idea that normative properties play different roles than their descriptive counterparts in arguing for their distinctness, cf. Enoch: 20 I I, p. I 40. 
property $\mathrm{N}$ will have the same higher-order properties as its non-normative disjunctive counterpart $\mathrm{D}$, on the basis of these properties having the same grounds. A difference in higher-order properties thus presupposes distinctness rather than establishing it.

This problem is analogous to the grounding problem regarding coinciding entities. In that context, the pluralist's appeal to Leibniz's Law is problematic, given that the differences in properties amongst coinciding objects that the pluralist invokes lack a ground, due to the fact that coinciding objects share the same supervenience base. Likewise, the sameness of grounds of these necessarily co-extensive properties makes it difficult to explain how they could differ in higher-order properties. The task is thus to explain what accounts for the differences amongst them in terms of how these properties are grounded, rather than to presuppose there being differences amongst them and then appealing to these differences to argue for their distinctness. As we will see below, the differences that separate these properties cannot be explained by identifying different grounds (since they share the very same grounds), but instead by identifying different grounding relations via which these properties are grounded. ${ }^{20}$

\section{Interlude on reduction}

The grounding argument threatens to rule out irreducibly normative properties insofar as it establishes that for every normative property $\mathrm{N}$ there is a hyperintensionally equivalent descriptive property $\mathrm{D}$. The reducibility of normative properties consists in there being descriptive properties to which they are identical. This means that the reduction of the normative is not a matter of the 'vertical' relation between $\mathrm{N}$ and its various grounds, but is rather concerned with the 'horizontal' relation between $\mathrm{N}$ and $\mathrm{D}$. The normative property $\mathrm{N}$ is not reduced to its grounds (whether taken individually or collectively). Instead, a property identity is established between $\mathrm{N}$ and the disjunctive property $\mathrm{D}$ that has the very same grounds as $\mathrm{N}$.

It might be objected that reduction is an asymmetrical notion, i.e. if $\mathrm{x}$ is reduced to $y$ then $y$ cannot be reduced to $x$, yet that property identities are symmetrical. The asymmetry of reduction is, however, compatible with construing reduction as establishing property identities. This is because reducing the normative to the descriptive amounts to establishing that for every normative property there is a descriptive property to which it is identical but that it is not the case that every descriptive property has a corresponding normative property to which it is identical. The asymmetry is to be found at the level of classes of properties,

\footnotetext{
${ }^{20} \mathrm{~A}$ further similarity to the case of coinciding objects is that the pluralist needs to show that the differences in higher-order properties are genuine differences that cannot be explained away by means of predicational shifts (as Streumer attempts to do by invoking different modes of presentation, cf. Streumer: 2013, p. 327).
} 
not at the level of the particular properties between which property identities are established. Although the relation between a particular normative property $\mathrm{N}$ and its descriptive disjunctive counterpart $\mathrm{D}$ is a symmetrical identity relation, the relation between the class of normative properties and the class of descriptive properties is asymmetric since the former will be a proper subset of the latter.

A further objection to this understanding of reduction is that property identities are metaphysically uninteresting. Schroeder, for instance, has claimed that "it makes reductive views out not to really be theses of metaphysics at all, but only in the philosophy of language or epistemology" (cf. Schroeder: 2007, p. 64). The thought seems to be that identities are trivial from a metaphysical point of view since everything is identical to itself and to nothing else, such that a property identity does not tell us anything about the property itself but only something about language, namely that we pick out the same property by means of different expressions, i.e. we learn that certain expressions are co-referring.

This critique, however, is misguided since it is a substantive metaphysical matter whether the property identities required for the reducibility of normative to descriptive properties can be established.

First, there is the (relatively uncontroversial) issue of the closure conditions that apply to descriptive properties. For there to be a reductive identity, the disjunctive property $\mathrm{D}$ has to be a descriptive property, which means that the reductive identity presupposes certain closure conditions. Since it is a non-trivial matter which property-forming operations preserve the properties required for classifying as a descriptive property, the reductive identity will not be trivial but metaphysically substantive.

Second, as we will see in the next section, there is the issue of the nature of the grounding relation giving rise to normative properties, in particular whether the disjunctive property $\mathrm{D}$ is grounded in the very same way (that is, via the same grounding relation) as the normative property $\mathrm{N}$. If the relevant closure conditions hold and if the same grounding relation is at issue, then $\mathrm{D}$ and $\mathrm{N}$ will be identical, where this is a substantive metaphysical claim that is established on the basis of metaphysical reasoning that reveals something important about the nature of normative properties, namely how such properties are grounded, and not merely something about how we pick out properties.

Third, as we have just seen, the reducibility of the normative is not a claim about a particular property identity but a claim about an entire class of properties. This means that, even if particular property identities should not be metaphysically interesting, it would nevertheless be a metaphysically substantive matter that all normative properties are identical to descriptive properties but not vice versa, i.e. that the former constitute a proper subset of the latter. ${ }^{2 I}$

\footnotetext{
${ }^{21}$ For a more detailed treatment of the relation between reduction and grounding cf. "Grounding, reduction and analysis" (Bader: manuscript).
} 


\section{Normative grounding}

Instead of rejecting hyperintensional equivalence as the criterion of property identity, one should refine the understanding of hyperintensional equivalence and distinguish the different types of grounding relations that are involved in grounding the normative and the corresponding non-normative property. One thereby ensures that the two properties turn out to be hyperintensionally inequivalent after all. Whilst they neither differ in terms of their extensions across modal space, nor in terms of their grounds, one can nonetheless distinguish them in terms of the respective grounding relations that they involve.

In particular, whereas the normative property is normatively grounded, the disjunctive non-normative property is metaphysically grounded. Premise $I^{\prime}$ then becomes I*: 'normative properties are normatively grounded in non-normative properties', ${ }^{22}$ whereas premises $3^{\prime}$ and $4^{\prime}$ become $3^{*}$ : 'disjunctive properties are (individually) metaphysically grounded in their disjuncts' and $4^{*}$ : 'conjunctive properties are (collectively) metaphysically grounded in their conjuncts', from which one can no longer derive the existence of a hyperintensionally equivalent non-normative property corresponding to any given normative property.

Hyperintensional equivalence, accordingly, has to be understood as requiring not only sameness of grounds but also that the properties are grounded in the same ways, i.e. that the types of grounding relations that give rise to them are the same. This means that the theory that was sketched above needs to be supplemented by introducing a range of different grounding relations, such as metaphysical grounding $\left(\mathrm{g}_{\mathrm{M}}\right)$, normative grounding $\left(\mathrm{g}_{\mathrm{N}}\right)$, and nomological grounding $\left(\mathrm{g}_{\mathrm{C}}\right)$. The i-grounding set $\mathrm{g}_{\mathrm{i}}(\mathrm{F})$ does not have to contain basic grounders, i.e. it is not required that $\Gamma \in \mathcal{B}$ in order for $\Gamma \in \mathrm{g}_{\mathrm{i}}(\mathrm{F})$. Instead, it contains the (proper and improper) $\mathrm{i}$-basic grounds of $\mathrm{F}$ that are the ultimate grounds in $\mathrm{i}$ grounding chains giving rise to $\mathrm{F}$, i.e. $\mathrm{g}_{\mathrm{i}}(\mathrm{F})=\left\{\Gamma: \Gamma\right.$ grounds $_{\mathrm{i}} \mathrm{F} \wedge \neg \exists \Delta(\Delta \neq$ $\Gamma \wedge \Delta$ grounds $\left.\left._{\mathrm{i}} \Gamma\right)\right\}$, where every property is an improper ground of itself with respect to every grounding relation. ${ }^{23}$ Identity then requires not only that prop-

\footnotetext{
${ }^{22}$ Normative properties are normatively grounded in non-normative properties in the sense that every normative instantiation of a normative property will be such that some link in its grounding chain involves a normative grounding relation. Not every link connecting the normative to the non-normative needs to involve normative grounding since non-basic normative properties are metaphysically grounded in other normative properties (e.g. $\mathrm{N}_{\mathrm{I}} \vee \mathrm{N}_{2}$ is metaphysically grounded in its disjuncts). The restriction to normative instantiations is required because disjunctive properties such as $\mathrm{N} \vee \mathrm{D}$ might well be classified as normative, but can be metaphysically grounded in their non-normative disjuncts. When dealing with this kind of disjunctive property one has to distinguish between normative and non-normative instantiations. It is normatively instantiated if it obtains in virtue of $\mathrm{N}$ whereas it is non-normatively instantiated if it obtains in virtue of $\mathrm{D}$ (this is analogous to how a disjunctive property can be had intrinsically and/or extrinsically depending on whether it is had in virtue of its intrinsic and/or extrinsic disjuncts).

${ }^{23}$ In case normative laws should be metaphysically contingent and should admit of variation across modal space, one would have to specify not only the type of grounding relation but also
} 
erties are grounded in the same things but also that they are grounded in them via the same relations, such that (non-fundamental) properties $F$ and $G$ are identical iff $g_{i}(F)=g_{i}(G)$ for some grounding relation $g_{i}$. This allows some nonfundamental properties to be individuated in terms of (pluralities of) other (more fundamental) non-fundamental properties, generating a recursive structure that ultimately terminates in basic grounders, i.e. in compatible pluralities of fundamental properties, where fundamental properties are $\mathrm{i}$-basic with respect to every i-grounding chain.

This modification ensures that we take into consideration not only the relata of the grounding relation but also the nature of the grounding relation. Differences between (non-fundamental) properties still have to be groundingdifferences, yet this is no longer understood merely in terms of having different grounds but also in terms of being grounded in different ways. Since the relation connecting the grounds to what is grounded therein is a worldly matter, this refined condition satisfies the worldliness constraint. The differences between properties that it identifies are thus genuine distinctions in the world that are either due to different aspects of the world accounting for the different properties or due to the properties being grounded in different ways.

Normative properties and their disjunctive descriptive counterparts do not satisfy the conditions of hyperintensional equivalence once one distinguishes normative from metaphysical grounding. $\mathrm{N}$ and $\mathrm{D}$ will have different i-grounding sets, i.e. $\mathrm{g}_{\mathrm{i}}(\mathrm{N}) \neq \mathrm{g}_{\mathrm{i}}(\mathrm{D})$ for all grounding relations $\mathrm{g}_{\mathrm{i}} \cdot{ }^{24}$ Normative properties and their non-normative counterparts are thus distinct, not because they have different grounds, but because they are grounded in the same grounds in different ways, namely via different types of grounding relations. This holds even if metaphysical and normative modality should turn out to be co-extensive, i.e. even if there is a unique set of norms that holds in all metaphysically possible worlds. Non-reductionist versions of moral realism, accordingly, require a commitment to there being irreducibly distinct types of grounding relations. ${ }^{25}$

In particular, one has to think of the normative grounding relations as being governed by robust grounding principles. This is because it is not possible to make sense of $\Gamma$ normatively grounding $\mathrm{N}$ whilst metaphysically grounding $\mathrm{D}$ unless these are distinct grounding relations that operate in accordance with different principles. The differences between $\mathrm{N}$ and $\mathrm{D}$ are to be explained in terms of the differences in the relations giving rise to them. Normative grounding principles thus need to be distinct from those involved in metaphysical grounding and need

relativise grounds to sets of worlds (or better, to sets of norms), so that grounding sets have the following form: $\mathrm{g}_{\mathrm{N}}(\mathrm{F})=\left\{\Gamma_{\mid \mathrm{w}_{\mathrm{I}} \ldots \mathrm{w}_{\mathrm{i}}}, \Delta_{\mid \mathrm{w}_{\mathrm{j}} \ldots \mathrm{w}_{\mathrm{k}}}, \Lambda_{\mid \mathrm{w}_{\mathrm{l}} \ldots \mathrm{w}_{\mathrm{n}}}\right\}$.

${ }^{24}$ They will only have the same grounding set with respect to the generic grounding relation $g_{V}$ which is constructed by taking the transitive closure of the various specific grounding relations.

${ }^{25}$ Dispositional essentialists who consider nomological and metaphysical possibility to coincide can likewise distinguish dispositional properties from their disjunctive categorical counterparts insofar as the former are nomologically grounded, whilst the latter are metaphysically grounded. 
to be prior to the grounding facts that they govern. ${ }^{26}$ These grounding principles cannot be mere descriptions of patterns of grounding facts but have to be doing substantive work and have to be appealed to in individuating grounding relations.

Normative properties are thus irreducible because they are grounded differently, where this does not involve being grounded in different things but, instead, being grounded via different grounding relations. This explanation satisfies the worldliness constraint, since there is something worldly that accounts for the distinctness of these properties, namely the different grounding relations that give rise to them. In this way, one can ensure that there are genuine worldly differences between them. The distinction between normative and non-normative properties, accordingly, classifies as a real rather than merely nominal distinction.

By accepting such different grounding relations, one can, moreover, explain why normative properties differ in kind from their disjunctive descriptive counterparts. The difference in the nature of the grounding relation generates differences in the kinds of property being grounded. There is thus no need to reject the closure principles. The account can, in this way, respect the fact that one cannot arrive at a normative property by simply putting together various non-normative properties. By disjoining and conjoining non-normative properties one only generates further non-normative properties. To end up with a normative property, one needs to do something else, namely bring in a normative grounding relation.

In this way, one can explain the heterogeneity of these properties, whilst at the same time allowing them to be grounded in the same base. Sameness of ground combined with difference in grounding relation explains both necessary co-extensiveness as well as distinctness and difference in kind. On the one hand, there is no need to posit any brute necessary connections, since the fact that they are necessarily co-extensive is explained in terms of them having a common ground. ${ }^{27}$ On the other hand, their radical heterogeneity is explained in terms of them being grounded via different types of grounding relations.

Relatedly, the commitment to normative grounding relations allows us to explain the irreducibility of normative properties on the basis of a commitment that has a good claim to constituting the "core metaphysical thesis of non-naturalism" and that genuinely classifies as "a substantial (and controversial) thesis about the metaphysics of the normative" (Dunaway: 2015, p. 645). That there is an irreducibly different normative grounding relation clearly is such a metaphysical thesis. The property distinctness is thus explicable in terms of the very feature that makes non-reductive or non-natural normative realism the distinctive and

\footnotetext{
${ }^{26}$ The situation is analogous to the grounding problem regarding coinciding entities. In the same way that different types of grounding relations can generate different properties out of the same base, a mereological pluralist who recognises different types of composition relations can explain how different objects can be generated out of the same parts.

${ }^{27}$ Normative grounding explains why they will be co-extensive in (at least) all normatively possible worlds. In case there should be a unique set of normative laws that holds in all of modal space, then they will also be necessarily co-extensive with respect to metaphysical modality.
} 
controversial theory that it is. In this way, the proposed account satisfies Dunaway's desideratum that the denial of the property identity follows from the core commitment of non-naturalism and thereby distinguishes it from other cases, such as the case of triangularity and trilaterality, in which necessarily co-extensive properties are distinct but in which no analogous non-naturalist metaphysical commitments are incurred.

A plurality of grounding relations thus allows us to explain the distinctness and heterogeneity of normative and descriptive properties in terms of a commitment that plausibly classifies as the metaphysical core of non-naturalism, whilst also explaining why these properties are necessarily co-extensive. This means that we have a conditional argument to the effect that one needs to countenance an irreducibly distinct normative grounding relation if one is to defend non-reductive moral realism against the grounding argument. ${ }^{28}$ In order to make plausible the existence of a plurality of grounding relations, one needs to develop a systematic theory of grounding principles that explains how they work and how they are to be individuated. In particular, one needs to give an account of what grounding principles there are that avoids a proliferation of different grounding relations. ${ }^{29}$

\section{Amalgamating normative grounds}

In constructing the disjunctive descriptive counterpart $\mathrm{D}$ one disjoins the conjunctive properties that are formed by conjoining the members of $\Gamma_{i}$ for every possible instantiation $\mathrm{n}_{\mathrm{i}}$ of $\mathrm{N}$. The disjunctive property $\mathrm{D}$ will only be hyperintensionally equivalent to $\mathrm{N}$, if the grounds of $\mathrm{N}$ satisfy an amalgamation condition that requires that compatible combinations of members of $g(N)$ are themselves members of $\mathrm{g}(\mathrm{N})$. That is, if $\Gamma_{\mathrm{I}}$ and $\Gamma_{2}$ are compatible (i.e. $\Gamma_{\mathrm{I}} \circ \Gamma_{2} \in \mathcal{B}$ ), then their combination also has to be one of the $\Gamma_{\mathrm{i}}$ such that they jointly ground $\mathrm{N}$. This is because a disjunction is grounded not only individually in (the basic grounders of) its disjuncts but also collectively when these are compatible.

The issue now is that it is not at all clear whether normative properties are always collectively grounded in this way. For instance, a determinate evaluative property, such as being valuable to degree $=5$, can be grounded in different compatible ways, such that something can have this property because of $\Gamma_{\mathrm{I}}$ as well as because of $\Gamma_{2} \cdot{ }^{30}$ Yet, these grounds do not collectively ground this evaluative

\footnotetext{
${ }^{28}$ Accepting different grounding relations implies accepting different sources of necessity. However, pace Fine: 2005 pp. 258-259, this does not mean that normative necessity cannot be understood as a relative modality.

${ }^{29}$ Cf. "Two levels of good-making" (Bader: manuscript) for discussion of these issues.

${ }^{30}$ Whether this is possible depends on how value bearers are construed. If property instantiations or states-of-affairs constitute value bearers, then the claim that $\mathrm{x}$ is good because $\mathrm{x}$ is $\mathrm{F}$ is to be construed as saying that x's being $\mathrm{F}$ is good, i.e. not that $\mathrm{x}$ both has the property of being good and the property of being $\mathrm{F}$ whereby the latter grounds the former, but rather that $\mathrm{x}$ is $\mathrm{F}$ where x's being $F$ is the ground of the value of x's being F. Accordingly, there will be two different value
} 
property. ${ }^{3 \mathrm{I}}$ What corresponds to the grounds taken together is the object's (nonbasic) value of degree $=$ Io. That is, the object has basic value due to each ground but not due to the grounds taken collectively. Instead, only non-basic value corresponds to the grounds taken together. Imposing the amalgamation constraint on normative properties ensures that the combination of the two grounds also classifies as a ground of basic value, which in this case leads to overgenerating value. To avoid this problematic consequence one needs to distinguish between basic value that is normatively grounded in $\Gamma_{\mathrm{I}}$ as well as in $\Gamma_{2}$, and metaphysically grounded non-basic value that corresponds to their combination, thereby avoiding double-counting.

If the grounds of $\mathrm{N}$ do not amalgamate, then this precludes premise $3^{\prime}$ from playing its intended role, leading to a difference in grounds. The grounds of $\mathrm{N}$ will then be a proper subset of those of $\mathrm{D}$, since the latter will also include the amalgamated grounds. Moreover, given that the grounding set for $\mathrm{N}$ fails to satisfy the amalgamation condition that has to be satisfied by any metaphysically grounded property, there will not be any metaphysically grounded property and hence not any descriptive property that has the same grounds as N. Since reduction only works via metaphysical grounding relations (since they are required to preserve the base properties in question), $\mathrm{N}$ will be irreducible.

Accordingly, it might seem that there is a different way of avoiding the grounding argument that does not appeal to normative grounding relations, namely by pointing out that non-amalgamating normative properties are irreducibly normative because there are no descriptive properties that have the same grounds. This difference between descriptive and non-amalgamating (basic) normative properties, however, can only arise because of a difference in grounding relations.

Amalgamation can be established for metaphysical grounding on the basis of $\wedge$-idempotency, i.e. the fact that $F \wedge F=F$, together with the rule for conjoining properties. $\wedge$-idempotency holds if $\Gamma \in \mathrm{g}(\mathrm{F})$ iff $\Gamma \in \mathrm{g}(\mathrm{F} \wedge \mathrm{F})$. This is only the case if metaphysically grounded properties satisfy the amalgamation constraint. Otherwise, there could be compatible grounders of F, e.g. $\mathrm{G}$ and $\mathrm{H}$, without the conjunctive ground $\mathrm{G} \circ \mathrm{H}$ being a grounder of $\mathrm{F}$, but this would imply that the conjunctive ground would be in the grounding set of $F \wedge F$. Contrary to $\wedge$ idempotency, $\mathrm{F}$ and $\mathrm{F} \wedge \mathrm{F}$ would then have different grounding sets, i.e. $\mathrm{g}(\mathrm{F})=$

bearers, rather than one thing that is valuable both because of $\Gamma_{1}$ and because of $\Gamma_{2}$.

Moreover, $\mathrm{D}$ and $\mathrm{N}$ will not even be necessarily co-extensive, since, although $\mathrm{D}$ will be instantiated whenever $\mathrm{N}$ will be instantiated, they will not be instantiated by the same entity. $\mathrm{D}$ will be a property of $\mathrm{x}$, whereas $\mathrm{N}$ will be a property of the property instantiation. One can appeal to descriptive properties of property instantiations to construct a disjunctive descriptive property $\mathrm{D}^{*}$ that is necessarily co-extensive with $\mathrm{N}$, but this property will not be hyperintensionally equivalent since $\mathrm{N}$ will be grounded in descriptive properties of $\mathrm{x}$ whereas $\mathrm{D}^{*}$ will be grounded in descriptive properties of property instantiations.

${ }^{3}$ S Some normative properties are normatively grounded both individually and collectively, namely certain holistic goods, such that $\Gamma_{1}$ and $\Gamma_{2}$ both individually and collectively give rise to basic value (though possibly to different degrees of value). 
$\{\mathrm{G}, \mathrm{H}\}$ but $\mathrm{g}(\mathrm{F} \wedge \mathrm{F})=\{\mathrm{G}, \mathrm{H}, \mathrm{G} \circ \mathrm{H}\}$, and would hence be distinct.

Since the only metaphysical ground of a basic normative property $\mathrm{N}$ is the improper ground, namely $\mathrm{N}$ itself, it follows that $\mathrm{g}_{\mathrm{M}}(\mathrm{N})=\mathrm{g}_{\mathrm{M}}(\mathrm{N} \wedge \mathrm{N})=\{\mathrm{N}\}$. $\wedge$-idempotency is hence validated for normatively grounded properties even if their normative grounds do not satisfy the amalgamation condition. There is thus only one way in which a property can have non-amalgamating grounds without violating $\wedge$-idempotency, namely insofar as the grounding relation involved in $\mathrm{N} \wedge \mathrm{N}$, which is metaphysical, is distinct from the grounding relation involved in N. This means that only basic normative properties, i.e. properties that are normatively grounded, can be non-amalgamating. Non-basic normative properties that are metaphysically grounded in other normative properties have to satisfy the amalgamation constraint.

$\mathrm{N}$ and $\mathrm{D}$ can thus be hyperintensionally inequivalent due to having different grounds, insofar as the grounding set of $\mathrm{D}$ satisfies amalgamation whereas that of $\mathrm{N}$ does not (where this difference is only possible because these properties are grounded via different grounding relations). In this way, one can establish the irreducibility of non-amalgamating normative properties. Yet when it comes to normative properties that have incompatible grounds, as well as normative properties the grounds of which are compatible but where $\mathrm{N}$ is also grounded in the combination of those grounds (as happens in the case of certain holistic goods), the grounding sets of $\mathrm{N}$ and $\mathrm{D}$ do not differ in this way. To establish the distinctness of such properties, one has to appeal not to a difference in grounds but a difference in grounding relations.

\section{Conditional normative grounds}

We have so far been making use of a broad notion of grounding that includes conditions (as well as modifiers) ${ }^{32}$, such that the grounds constitute a necessitating base. Yet, in certain cases we need to draw more fine-grained distinctions by distinguishing sources (or grounds narrowly construed) from conditions on which the grounding relation merely depends. This is because certain normative properties are only conditionally grounded, requiring enablers to be present and/or disablers to be absent. ${ }^{33}$ For instance, according to Kant, happiness is only good when had by someone who has a good will.

Drawing such distinctions requires us to include additional structure into the grounding set. Since we have to make room for conditions on grounding relations, we have to work with ground $s_{C}$ where an unconditional grounding relation obtains if $\mathrm{C}=\emptyset$. The grounding set of a property $\mathrm{F}$ can be understood as follows: $\mathrm{g}(\mathrm{F})=\left\{\langle\Gamma, \mathrm{C}\rangle: \Gamma \in \mathcal{B} \wedge\right.$ grounds $\left._{\mathrm{C}} \mathrm{F}\right\}$. Hyperintensional equivalence then

\footnotetext{
${ }^{32}$ The discussion in this section applies mutatis mutandis to the case of modification.

${ }^{33}$ Cf. "Conditional grounding" (Bader: manuscript) for an account of conditional grounding, and Bader: 2016 for an application of conditionality and modification to the normative realm.
} 
requires that properties have the same grounds subject to the same conditions. This means that properties can be distinct, despite having the same necessitation base, due to having differently structured grounding sets, i.e. the elements of the necessitation base can differ in terms of whether they are assigned a groundingrole or a condition-role

Premise $\mathrm{I}^{\prime}$ in the grounding argument should thus be replaced by $\mathrm{I}^{\prime}$-COND: 'some normative properties are conditionally grounded in non-normative properties' as well as I'-UNCOND: 'some normative properties are unconditionally grounded in non-normative properties'. Conjunctive grounding, however, is unconditional. This means that premise $4^{\prime}$ is to be construed as $4^{\prime}$-UNCOND: 'all conjunctive properties are (collectively) unconditionally grounded in their conjuncts', which implies that this premise can no longer play its intended role in the argument. In particular, it will not be possible to construct a disjunctive descriptive counterpart for a conditionally grounded normative property.

Every instantiation $n_{i}$ of such a property will be conditionally grounded in some ground $\Gamma_{\mathrm{i}}$, such that this grounding relation only obtains because condition $C_{i}$ is satisfied. As a result, $g(N)=\left\{\left\langle\Gamma_{I}, C_{I}\right\rangle, \ldots,\left\langle\Gamma_{n}, C_{n}\right\rangle\right\}$. One can construct two different descriptive properties. On the one hand, one can disjoin the conjunctive properties that result from combining the members of $\Gamma_{i}$, i.e. $\mathrm{D}=\bigvee\left\{\wedge \Gamma_{\mathrm{i}}\right.$ for all $\left.\Gamma_{\mathrm{i}} \in \mathrm{g}(\mathrm{N})\right\}$. This property will have the same grounds as $\mathrm{N}$ but will not have the conditions built in, and as such will not even be extensionally equivalent to $\mathrm{N}$, since $\mathrm{D}$ will be had whereas $\mathrm{N}$ will be lacked whenever the conditions are not satisfied (assuming that $\mathrm{N}$ is not overgrounded in those cases). On the other hand, one can disjoin the conjunctive properties that result from combining the members of $\Gamma_{i}$ as well as $C_{i}$, i.e. $D=\bigvee\left\{\wedge\left(\Gamma_{i} \wedge C_{i}\right)\right.$ for all $\left.\left\langle\Gamma_{\mathrm{i}}, \mathrm{C}_{\mathrm{i}}\right\rangle \in \mathrm{g}(\mathrm{N})\right\}$. This property will be extensionally equivalent to $\mathrm{N}$, yet will be hyperintensionally distinct since the members of $g(D)$ will not be structured in the same way as $\mathrm{g}(\mathrm{N})$.

What is required for hyperintensional equivalence is that $\mathrm{g}(\mathrm{D})$ contains the grounds of $\mathrm{N}$ where these grounds are subject to the very same conditions. Since conjunctive grounding is unconditional, the disjuncts of $\mathrm{D}$ will not be conjunctive properties, which means that the closure conditions specified in premise $2^{\prime}$ are not applicable. Hyperintensional equivalence thus requires not only that descriptive properties are closed under infinitary conjunction and disjunction but also that if $\Gamma$ and $\mathrm{C}$ are descriptive, then a property conditionally grounded in $\Gamma$ subject to condition $\mathrm{C}$ will also be descriptive.

The problem now is that not every (consistent) combination of descriptive grounds and conditions grounds a descriptive property. Conditional grounding relations presuppose a mechanism of enabling or disabling that explains how the non-satisfaction of the conditions undermines the grounding relation in question. Whilst there are conditionally grounded descriptive properties, there is no guarantee that there will be conditionality mechanisms operating at the descrip- 
tive level that apply whenever a normative property $\mathrm{N}$ is conditionally grounded. The reductive realist thus has to establish on a case by case basis that descriptive conditionality mechanisms can be identified whenever normative conditionality mechanisms apply.

This means that, although one can always construct a descriptive property by conjoining and disjoining various descriptive properties, there is no guarantee that one can always construct a conditionally grounded descriptive property. $\mathrm{Hy}-$ perintensional equivalence can thus, in principle, only be established on the basis of premise I'-UnCOND but not premise I'-COND. This means that the grounding argument only shows that some normative properties (namely all those that are unconditionally grounded) are such there is a non-normative property that is hyperintensionally equivalent, which, together with the hyperintensional equivalence criterion for property identity, in turn only establishes the restricted conclusion that some normative property (namely all those that are unconditionally grounded) are identical to non-normative properties.

Considerations of conditionality imply that the grounding argument by itself only establishes a restricted reducibility claim. This conclusion is still problematic for the non-reductive moral realist and a commitment to grounding pluralism is required to establish irreducibility across the board. In addition, it can be argued that (in those cases where conditionality cannot be motivated at the descriptive level) the conditionality of normative properties itself presupposes a commitment to grounding pluralism, since it is only by bringing in normative grounding principles that one can explain why $\Gamma$ fails to conditionally ground a descriptive property $\mathrm{D}$ yet does conditionally ground a normative property $\mathrm{N}$.

\section{Ethical idlers?}

Bringing in normative grounding relations that are governed by normative grounding principles also allows us to defuse the objection that "it is hard to see how the further properties could be of any ethical significance. Are we supposed to take seriously someone who says, 'I see that this action will kill many and save no-one, but that is not enough to justify my not doing it; what really matters is that the action has an extra property that only ethical terms are suited to pick out'? In short, the extra properties would [be] ethical 'idlers'." (Jackson: I998, p. I27)

In response we can note that the non-normative features are ethically significant only on the basis that they ground normative properties and that their doing so is dependent on the normative grounding principles. What matters is that the action has the relevant non-normative properties that give rise to the normative property in question, e.g. those that justify the action. Precisely which non-normative properties are relevant and account for the normative status of the action is determined by the normative grounding principles. These principles select amongst the vast array of non-normative features those that are relevant, 
by making it the case that these features ground normative properties.

This means that non-normative features only have ethical significance when they fall under a normative grounding principle. They are ethically significant because they are imbued with normative significance by the grounding principle. Since the grounding principle is precisely what gets us to the normative property, we can see that what it is for non-normative properties to have ethical significance is to give rise to normative properties. They are hence not ethical idlers but are necessary for ethical significance. In other words, the significance and justificatory force of the non-normative features lies precisely in the fact that they ground normative properties. A full explanation of the normative status of the action in this way cannot remain at the non-normative level, but has to invoke grounding principles and go to the normative level.

That the significance of non-normative properties is to be explained in this way is particularly clear in cases in which they only conditionally ground normative properties, requiring enablers to be present and/or disablers to be absent. These cases show that the properties that are selected at the non-normative level do not by themselves suffice for grounding normative properties. Since they are only conditional grounds, they only have normative significance conditionally. Accordingly, one has to appeal to the normative level to identify and explain the conditions under which these features do in fact give rise to normative properties, namely when the enabling/disabling conditions are satisfied. This means that these non-normative features are only to be selected under certain conditions, where the selection is determined by the normative grounding principles.

Although one can fully specify in descriptive terms the necessitating base of the goodness, the non-normative features on which the goodness supervenes need to be distinguished into those things that are of ethical significance and those that merely constitute the conditions under which the former are of ethical significance. The explanation as to why these conditions need to be satisfied is precisely because they are required for the (conditional) grounds to actually give rise to normative properties and thereby acquire ethical significance. One can, accordingly, only explain the relevance of these conditions in terms of their effect on the grounding of normative properties. This means that normative properties turn out to be explanatorily indispensable.

Far from being idle, normative properties as well as their corresponding normative grounding principles are necessary for imbuing non-normative features with normative significance and for selecting the relevant non-normative features in the relevant circumstances.

\section{Io Conclusion}

The standard reply to the supervenience argument is thus insufficient for vindicating non-reductive realism due to the grounding argument that functions as 
its hyperintensional analogue. What makes room for a non-reductive account of normative properties is that a normative grounding relation gives rise to these properties which is distinct from the metaphysical grounding relation giving rise to their disjunctive descriptive counterparts. It is thus possible to accept that the normative is grounded in the non-normative, without ending up with a reductionist view, as long as the grounding relation is construed as involving normative rather than metaphysical grounding. Some will take this result as yet further reason to reject non-reductive versions of moral realism, whilst others will consider the idea of irreducibly distinct normative grounding relations to be a fruitful line of inquiry that is worthy of being pursued further. Yet, what is clear is that the fate of non-reductive realism is inextricably tied up with there being irreducibly different grounding relations. ${ }^{34}$

\section{References}

[I] BADER, R. M. Supervenience and infinitary property-forming operations. Philosophical Studies I60 (2012), 4I 5-423.

[2] Bader, R. M. Conditions, modifiers, and holism. In Weighing Reasons, E. Lord and B. Maguire, Eds. Oxford University Press, 2016, pp. 27-55.

[3] Dunaway, B. Supervenience arguments and normative non-naturalism. Philosophy and Phenomenological Research 9I, 3 (2015), 627-655.

[4] Enoch, D. Taking Morality Seriously: A Defense of Robust Realism. Oxford University Press, 20 I I.

[5] Fine, K. Modality and Tense: Philosophical Papers. Oxford University Press, 2005 .

[6] Glanzberg, M. Supervenience and infinitary logic. Nô̂s 35, 3 (200 I), 4I9-439.

[7] Jackson, F. From Metaphysics to Ethics. Oxford University Press, I 998.

[8] KIM, J. Supervenience and Mind. Cambridge University Press, I993.

[9] Majors, B. Moral discourse and descriptive properties. Philosophical Quarterly 55 (2005), 475-494.

\footnotetext{
${ }^{34}$ For helpful comments and discussions I would like to thank audiences at Oxford, the metaethics workshop at Chapel Hill, and Kyoto. I am also grateful to Selim Berker, Neil Mehta, Billy Dunaway, Chris Blake-Turner, Barry Maguire, Derek Haderlie, Dan Fogal, Tobias Wilsch, as well as two anonymous referees.
} 
[io] McPherson, T. Ethical non-naturalism and the metaphysics of supervenience. Oxford Studies in Metaethics 7 (201 2), 207-245.

[i I] Scanlon, T. M. Being Realistic about Reasons. Oxford University Press, 2014 .

[i 2] Schroeder, M. Slaves of the Passions. Oxford University Press, 2007.

[i3] Shafer-Landau, R. Moral Realism: A Defence. Oxford University Press, 2003.

[14] Streumer, B. Are there irreducibly normative properties? Australasian Journal of Philosophy 86, 4 (2008), 537-56I.

[I5] Streumer, B. Why there really are no irreducibly normative properties. In Thinking about reasons: Themes from the philosophy of Jonathan Dancy, D. Bakhurst, B. Hooker, and M. Little, Eds. Oxford University Press, 2013 , pp. 31 $10-334$.

[i6] Suikkanen, J. Non-naturalism: The Jackson challenge. Oxford Studies in Metaethics 5 (2010), 87-1 I0.

[i7] van Cleve, J. Supervenience and closure. Philosophical Studies 58 (I990), $225-238$. 